\title{
HERMITIAN JACOBI FORMS AND $U(p)$ CONGRUENCES
}

\author{
OLAV K. RICHTER AND JAYANTHA SENADHEERA
}

(Communicated by Ken Ono)

Abstract. We introduce a new space of Hermitian Jacobi forms, and we determine its structure. Moreover, we characterize $U(p)$ congruences of Hermitian Jacobi forms, and we discuss an explicit example.

\section{InTRODUCTION AND STATEMENT OF MAIN RESUltS}

Jacobi forms appear naturally in various areas of mathematics and physics, and they connect different types of automorphic forms. In particular, they occur as Fourier-Jacobi coefficients of Siegel modular forms of degree 2, a fact that figured prominently in the solution of the Saito-Kurokawa conjecture (see [2, 8, 15, 17, 29,).

Hermitian modular forms are generalizations of Siegel modular forms, and Hermitian Jacobi forms occur as Fourier-Jacobi coefficients of Hermitian modular forms of degree 2. Haverkamp [10 11] systematically studied Hermitian Jacobi forms, and [5, 7, 23. contributed further to the theory of such forms.

The usual heat operator is an important device in the study of classical Jacobi forms (see, for example, [3,4,8]), and its action on Jacobi forms can be "corrected" so that Jacobi forms of weight $k$ are mapped to Jacobi forms of weight $k+2$ (see [22]). The heat operator

$$
L_{m}:=\frac{1}{(2 \pi i)^{2}}\left(8 \pi i m \frac{\partial}{\partial \tau}-4 \frac{\partial^{2}}{\partial w \partial z}\right)
$$

is a natural tool in the theory of Hermitian Jacobi forms, and it plays a vital role in Section 3 of this paper. Equation (3.7) of 14 implicitly gives an action of $L_{m}$ on Hermitian Jacobi forms, but unfortunately, (3.7) of 14 is not quite correct. In fact, the action of $L_{m}$ on the Hermitian Jacobi forms in [5, 6, 10, 23, cannot be "corrected" as in the case of classical Jacobi forms, and one needs a different notion of Hermitian Jacobi form.

In this paper, we introduce the more general space $J_{k, m}^{\delta}$ of Hermitian Jacobi forms over $\mathbb{Q}(i)$ of weight $k$, index $m$, and parity $\delta= \pm$, and we set $J_{k, m}:=$ $J_{k, m}^{+} \oplus J_{k, m}^{-}$(see Definition 2.1). A direct computation shows that if $\phi \in J_{k, m}^{\delta}$, then

$$
L_{m}(\phi)=\frac{(k-1) m}{3} E_{2} \phi+\widehat{\phi}
$$

where $E_{2}$ is the usual quasimodular Eisenstein series, and where $\widehat{\phi} \in J_{k+2, m}^{-\delta}$. The fact that $\phi$ and $\widehat{\phi}$ in (1.2) are Hermitian Jacobi forms of different parities explains the need for our Definition 2.1

Received by the editors June 11, 2014.

2010 Mathematics Subject Classification. Primary 11F50; Secondary 11F33.

The first author was partially supported by Simons Foundation Grant \#200765. 
We now introduce notation necessary to state our main results. Throughout, $k$ is an even integer, and $M_{k}$ and $S_{k}$ are the weight $k$ spaces of elliptic modular forms and cusp forms, respectively. We denote the Taylor coefficients of a Hermitian Jacobi form of parity $\delta$ by $\chi_{\mu, \nu}^{\delta}$ (see Section 2.3), and $\zeta_{\mu, \nu}^{\delta}$ are the combinations of Taylor coefficients in Proposition 2.7. Our first result gives the structure of Hermitian Jacobi forms over $\mathbb{Q}(i)$ of index 1 . More specifically, it asserts that $J_{k, 1}$ is a free module of rank 4 over the ring of modular forms, where a set of generators is given by the Hermitian Jacobi forms $\phi_{4,1}^{+}, \phi_{6,1}^{-}, \phi_{8,1}^{+}$, and $\phi_{10,1}^{+, \text {cusp }}$ (defined in (2.5) and Lemma 2.4).

Theorem 1.1. If $k \equiv 0(\bmod 4)$, then both linear maps

$$
\begin{aligned}
\zeta: J_{k, 1} & \rightarrow M_{k} \oplus S_{k+2} \oplus S_{k+2} \oplus S_{k+4}, \\
\phi & \mapsto\left(\chi_{0,0}^{+}, \zeta_{1,1}^{+}, \chi_{2,0}^{-}, \quad \zeta_{2,2}^{+}-12 \chi_{4,0}^{+}\right)
\end{aligned}
$$

and

$$
\begin{aligned}
\eta: M_{k-4} \oplus M_{k-6} \oplus M_{k-8} \oplus M_{k-10} & \rightarrow J_{k, 1} \\
(e, f, g, h) & \mapsto\left(e \phi_{4,1}^{+}+f \phi_{6,1}^{-}+g \phi_{8,1}^{+}, h \phi_{10,1}^{+, c u s p}\right)
\end{aligned}
$$

are isomorphisms.

If $k \equiv 2(\bmod 4)$, then both linear maps

$$
\begin{aligned}
\zeta: J_{k, 1} & \rightarrow M_{k} \oplus S_{k+2} \oplus S_{k+2} \oplus S_{k+4}, \\
\phi & \mapsto\left(\chi_{0,0}^{-}, \quad \zeta_{1,1}^{-}, \chi_{2,0}^{+}, \quad \zeta_{2,2}^{-}-12 \chi_{4,0}^{-}\right)
\end{aligned}
$$

and

$$
\begin{aligned}
\eta: M_{k-4} \oplus M_{k-6} \oplus M_{k-8} \oplus M_{k-10} & \rightarrow J_{k, 1}, \\
(e, f, g, h) & \mapsto\left(h \phi_{10,1}^{+, \text {cusp }}, e \phi_{4,1}^{+}+f \phi_{6,1}^{-}+g \phi_{8,1}^{+}\right)
\end{aligned}
$$

are isomorphisms.

Theorem 1.1 allows us to investigate congruences and filtrations of Hermitian Jacobi forms of index 1 . We introduce more necessary notation. Throughout, $p \geq 5$ is a prime. Let $\mathbb{Z}_{(p)}:=\mathbb{Z}_{p} \cap \mathbb{Q}$ be the local ring of $p$-integral rational numbers, $J_{k, 1}^{\delta}\left(\mathbb{Z}_{(p)}\right)$ the space of forms in $J_{k, 1}^{\delta}$ that have $p$-integral rational coefficients, $\Omega$ the filtration of a Hermitian Jacobi form in $J_{k, 1}^{\delta}\left(\mathbb{Z}_{(p)}\right)$ (see Definition [3.4), and $U(p)$ the following analog of Atkin's $U$-operator:

$$
\left(\sum_{\substack{n \in \mathbb{Z}, r \in \mathcal{O}^{\#} \\ n-|r|^{2} \geq 0}} c(n, r) q^{n} \zeta^{\bar{r}}\left(\zeta^{\prime}\right)^{r}\right) \mid U(p):=\sum_{\substack{n \in \mathbb{Z}, r \in \mathcal{O}^{\#} \\ n-|r|^{2} \geq 0 \\ p \mid 4\left(n-|r|^{2}\right)}} c(n, r) q^{n} \zeta^{\bar{r}}\left(\zeta^{\prime}\right)^{r},
$$

where here (and in the following) $q:=e^{2 \pi i \tau}, \zeta:=e^{2 \pi i z}, \zeta^{\prime}:=e^{2 \pi i w}$, and $\mathcal{O}^{\#}:=$ $\frac{1}{2} \mathbb{Z}[i]$ is the inverse different of $\mathcal{O}:=\mathbb{Z}[i]$. Finally, for convenience, we write $L:=L_{1}$. Our next theorem provides a criterion for the existence of $U(p)$ congruences of Hermitian Jacobi forms of index 1.

Theorem 1.2. Let $\phi \in J_{k, 1}^{\delta}\left(\mathbb{Z}_{(p)}\right)$ such that $\phi \not \equiv 0(\bmod p)$. If $p>k$, then

$$
\Omega\left(L^{p+2-k}(\phi)\right)=\left\{\begin{aligned}
2 p+4-k, & \text { if } \phi \mid U(p) \neq 0(\bmod p), \\
p+5-k, & \text { if } \phi \mid U(p) \equiv 0(\bmod p) .
\end{aligned}\right.
$$


Recall that $U(p)$ congruences of elliptic modular forms have applications in the context of traces of singular moduli and class equations (see [1,9, 18]). It would be interesting to see if $U(p)$ congruences for Hermitian Jacobi forms also find further applications.

The paper is organized as follows. In Section 2.1, we give a new definition of Hermitian Jacobi forms over $\mathbb{Q}(i)$. In Section 2.2, we discuss the theta decomposition of Hermitian Jacobi forms. In Section 2.3, we prove Theorem 1.1, In Section 3.1, we explore congruences of Hermitian Jacobi forms of index 1, and we provide the ingredients to prove Theorem 1.2 Finally, in Section 3.2 we present an explicit example to illustrate Theorem 1.2

Many of the results here have also been reported in the second author's University of North Texas doctoral dissertation 24.

\section{Hermitian Jacobi Forms over $\mathbb{Q}(i)$}

Hermitian Jacobi forms appear as Fourier-Jacobi coefficients of Hermitian modular forms of degree 2 over a complex quadratic field (see [7, 10]). In this paper, we restrict ourselves to the case where the complex quadratic field is the Gaussian number field $\mathbb{Q}(i)$. Throughout, $k$ and $m$ are nonnegative integers, and if $s \in \mathbb{C}$, then $\bar{s}$ denotes its complex conjugate.

2.1. A new definition. Recall from the introduction that $\mathcal{O}:=\mathbb{Z}[i]$ is the ring of Gaussian integers with inverse different $\mathcal{O}^{\#}:=\frac{1}{2} \mathbb{Z}[i]$. Let $\mathcal{O}^{\times}:=\{1,-1, i,-i\}$ be the group of units of $\mathcal{O}$ and $\Gamma(\mathcal{O}):=\left\{\epsilon M \mid \epsilon \in \mathcal{O}^{\times}, M \in S L_{2}(\mathbb{Z})\right\}$ be the Hermitian modular group.

Our following extension of Haverkamp's [10] notion of a Hermitian Jacobi form depends on a parity $\delta= \pm$.

Definition 2.1. A holomorphic function $\phi: \mathbb{H} \times \mathbb{C}^{2} \rightarrow \mathbb{C}$ is a Hermitian Jacobi form of weight $k$, index $m$, and parity $\delta$ if it satisfies the transformation laws

$$
\phi\left(\frac{a \tau+b}{c \tau+d}, \frac{\epsilon z}{c \tau+d}, \frac{\epsilon^{-1} w}{c \tau+d}\right)=\sigma(\epsilon) \epsilon^{k}(c \tau+d)^{k} e^{\frac{2 \pi i m c z w}{c \tau+d}} \phi(\tau, z, w)
$$

$$
\text { for all } \epsilon\left(\begin{array}{ll}
a & b \\
c & d
\end{array}\right) \in \Gamma(\mathcal{O}) \text {, }
$$

where $\sigma(\epsilon):=\left\{\begin{array}{lll}1 & \text { if } & \delta=+ \\ \epsilon^{2} & \text { if } & \delta=-\end{array}\right.$ and

$$
\phi(\tau, z+\lambda \tau+\mu, w+\bar{\lambda} \tau+\bar{\mu})=e^{2 \pi i m(\lambda \bar{\lambda} \tau+z \bar{\lambda}+\lambda w)} \phi(\tau, z, w), \text { for all }[\lambda, \mu] \in \mathcal{O}^{2} .
$$

Furthermore, one requires that $\phi$ has a Fourier series expansion of the form

$$
\phi(\tau, z, w)=\sum_{n=0}^{\infty} \sum_{\substack{r \in \mathcal{O}^{\#} \\ n m-|r|^{2} \geq 0}} c(n, r) q^{n} \zeta^{\bar{r}}\left(\zeta^{\prime}\right)^{r} .
$$

A Hermitian Jacobi form is called a cusp form if $c(n, r)=0$ unless $m n-|r|^{2}>0$. We denote the space of Hermitian Jacobi forms of weight $k$, index $m$, and parity $\delta$ by $J_{k, m}^{\delta}$, and the space of cusp forms in $J_{k, m}^{\delta}$ by $J_{k, m}^{\delta, c u s p}$. Finally, the space of Hermitian Jacobi forms of weight $k$ and index $m$ is defined by

$$
J_{k, m}:=J_{k, m}^{+} \oplus J_{k, m}^{-}=\left\{\left(\phi^{+}, \phi^{-}\right) \mid \phi^{+} \in J_{k, m}^{+}, \phi^{-} \in J_{k, m}^{-}\right\} .
$$


Remark 2.2. The space of Hermitian Jacobi forms in [5, 6, 10,23] coincides with the space of Hermitian Jacobi forms of positive parity, i.e., with $J_{k, m}^{+}$.

The next proposition follows exactly as Propositions 1.3 and 1.4 of [10] (see also Lemma 1 of [23]). We omit the proof, which is contained in [24].

Proposition 2.3. Let $\phi(\tau, z, w)=\sum c(n, r) q^{n} \zeta^{\bar{r}}\left(\zeta^{\prime}\right)^{r} \in J_{k, m}^{\delta}$. Then we have the following:

(i) The coefficient $c(n, r)$ depends only on $n m-|r|^{2}$ and on $r(\bmod m \mathcal{O})$.

(ii) If $\epsilon \in \mathcal{O}^{\times}$, then $\sigma(\epsilon) \epsilon^{k} c(n, r)=c(n, \bar{\epsilon} r)$.

(iii) If $m=1, k \equiv 0(\bmod 4)$, and $\delta=+$, then $c(n, r)$ depends only on $n-|r|^{2}$.

(iv) If $m=1, k \equiv 2(\bmod 4)$, and $\delta=-$, then $c(n, r)$ depends only on $n-|r|^{2}$.

(v) If $m=1$ and $k$ is odd, then $\phi=0$.

2.2. The theta decomposition. Haverkamp [10 establishes the so-called theta decomposition for Hermitian Jacobi forms of positive parity. Set

$$
\theta_{m, s}^{H}(\tau, z, w):=\sum_{\substack{r \in \mathcal{O}^{\#} \\ r \equiv s(\bmod m \mathcal{O})}} q^{|r|^{2} / m} \zeta^{\bar{r}}\left(\zeta^{\prime}\right)^{r} .
$$

Exactly as in [10], one finds that $\phi=\sum_{n, r} c(n, r) q^{n} \zeta^{\bar{r}}\left(\zeta^{\prime}\right)^{r} \in J_{k, m}^{\delta}$ has the theta decomposition

$$
\phi(\tau, z, w)=\sum_{s \in \mathcal{O} \# / m \mathcal{O}} h_{s}(\tau) \theta_{m, s}^{H}(\tau, z, w),
$$

where $h_{s}$ are certain vector-valued modular forms of weight $k-1$ (for more properties of $h_{s}$, see 24]). Note that we suppress the dependence on $\delta$ and we write $h_{s}$ instead of $h_{s}^{\delta}$.

For the remainder, we are only interested in Hermitian Jacobi forms of index 1. Observe that if $m=1$, then $\left\{0, \frac{1}{2}, \frac{i}{2}, \frac{1+i}{2}\right\}$ is a set of representatives for the set of cosets $\mathcal{O}^{\#} / m \mathcal{O}$. We now recall the theta decompositions for important examples of Hermitian Jacobi forms of positive parity. Consider the Jacobi theta function

$$
\theta_{a, b}(\tau, z):=\sum_{n \in \mathbb{Z}} e^{\pi i(a+n)^{2} \tau+2 \pi i(n+a)(z+b)} \quad(a, b \in \mathbb{R}),
$$

and its following specializations (theta constants):

$$
\begin{aligned}
& x:=\theta_{0,0}(\tau, 0)=1+2 \sum_{n=1}^{\infty} q^{\frac{n^{2}}{2}}, \\
& y:=\theta_{0, \frac{1}{2}}(\tau, 0)=1+2 \sum_{n=1}^{\infty}(-1)^{n} q^{\frac{n^{2}}{2}}, \\
& z:=\theta_{\frac{1}{2}, 0}(\tau, 0)=2 q^{\frac{1}{8}} \sum_{n=0}^{\infty} q^{\frac{n(n+1)}{2}} .
\end{aligned}
$$

It is well known (see, for example, [12]) that

$$
\begin{aligned}
& x^{4}=y^{4}+z^{4}, \\
& E_{4}=\frac{1}{2}\left(x^{8}+y^{8}+z^{8}\right), \\
& E_{6}=\frac{1}{2}\left(x^{4}+y^{4}\right)\left(x^{4}+z^{4}\right)\left(y^{4}-z^{4}\right),
\end{aligned}
$$


where $E_{k}(\tau):=1-\frac{2 k}{B_{k}} \sum_{n=1}^{\infty}\left(\sum_{d \mid n} d^{k-1}\right) q^{n}$ denotes the usual Eisenstein series.

Sasaki 23. provides the theta decomposition of several Hermitian Jacobi forms of index 1. In particular, he considers (up to normalization) the following Hermitian Jacobi forms $\phi_{k, 1}^{+} \in J_{k, 1}^{+}$for $k=4,8,12$ and $\phi_{10,1}^{+, \text {cusp }} \in J_{10,1}^{+, \text {cusp }}$ :

$$
\begin{aligned}
\phi_{4,1}^{+} & :=\frac{1}{2}\left(x^{6}+y^{6}\right) \theta_{1,0}^{H}+\frac{1}{2} z^{6}\left(\theta_{1, \frac{1}{2}}^{H}+\theta_{1, \frac{i}{2}}^{H}\right)+\frac{1}{2}\left(x^{6}-y^{6}\right) \theta_{1, \frac{1+i}{2}}^{H}, \\
\phi_{8,1}^{+} & :=\frac{1}{2}\left(x^{14}+y^{14}\right) \theta_{1,0}^{H}+\frac{1}{2} z^{14}\left(\theta_{1, \frac{1}{2}}^{H}+\theta_{1, \frac{i}{2}}^{H}\right)+\frac{1}{2}\left(x^{14}-y^{14}\right) \theta_{1, \frac{1+i}{2}}^{H}, \\
\phi_{12,1}^{+} & :=\frac{1}{2}\left(x^{22}+y^{22}\right) \theta_{1,0}^{H}+\frac{1}{2} z^{22}\left(\theta_{1, \frac{1}{2}}^{H}+\theta_{1, \frac{i}{2}}^{H}\right)+\frac{1}{2}\left(x^{22}-y^{22}\right) \theta_{1, \frac{i+1}{2}}^{H}, \\
\phi_{10,1}^{+, \text {cusp }} & :=\frac{1}{64} x^{6} y^{6} z^{6}\left(\theta_{1, \frac{1}{2}}^{H}-\theta_{1, \frac{i}{2}}^{H}\right) .
\end{aligned}
$$

Hermitian Jacobi forms of negative parity have not been studied rigorously in the literature, but they do arise via Fourier-Jacobi coefficients of Hermitian modular forms of degree 2 with certain characters (see [7). Hermitian Eisenstein series are examples of such Hermitian modular forms of degree 2. In particular, there exists such a Hermitian Eisenstein series of weight 6, whose first Fourier-Jacobi coefficient $\phi_{6,1}^{-}$is a Hermitian Jacobi form of negative parity, weight 6 , and index 1 . It is somewhat demanding to explicitly compute the Fourier series coefficients of the Hermitian Eisenstein series. We determined $\phi_{6,1}^{-}$via a different approach. We used SAGE [27] and the SAGE code written by Martin Raum to calculate some Fourier series coefficients of $\phi_{6,1}^{-}$, which allowed us to guess (and then prove) the correct theta decomposition of $\phi_{6,1}^{-}$.

Lemma 2.4. Set

$$
\phi_{6,1}^{-}:=h_{0} \theta_{1,0}^{H}+h_{\frac{1}{2}} \theta_{1, \frac{1}{2}}^{H}+h_{\frac{i}{2}} \theta_{1, \frac{i}{2}}^{H}+h_{\frac{1+i}{2}} \theta_{1, \frac{1+i}{2}}^{H},
$$

where

$$
\begin{aligned}
h_{0} & :=-\frac{1}{2}\left(x^{2}+y^{2}\right)\left(x^{8}-x^{6} y^{2}-x^{4} y^{4}-x^{2} y^{6}+y^{8}\right), \\
h_{\frac{1}{2}} & :=\frac{1}{2} z^{6}\left(z^{4}-2 x^{4}\right), \\
h_{\frac{i}{2}} & :=\frac{1}{2} z^{6}\left(z^{4}-2 x^{4}\right), \\
h_{\frac{1+i}{2}} & :=-\frac{1}{2}\left(x^{2}-y^{2}\right)\left(x^{8}+x^{6} y^{2}-x^{4} y^{4}+x^{2} y^{6}+y^{8}\right),
\end{aligned}
$$

and where $x, y$, and $z$ are as in (2.3). Then $\phi_{6,1}^{-} \in J_{6,1}^{-}$.

Proof. Consider $\psi_{12,1}^{+}:=-2 E_{4}^{2} \phi_{4,1}^{+}+\frac{15}{2} E_{4} \phi_{8,1}^{+}-\frac{9}{2} \phi_{12,1}^{+} \in J_{12,1}^{+}$, and let

$$
\psi_{12,1}^{+}=\hat{h}_{0} \theta_{1,0}^{H}+\hat{h}_{\frac{1}{2}} \theta_{1, \frac{1}{2}}^{H}+\hat{h}_{\frac{i}{2}} \theta_{1, \frac{i}{2}}^{H}+\hat{h}_{\frac{1+i}{2}} \theta_{1, \frac{1+i}{2}}^{H}
$$

be its theta decomposition. Then using (2.4) and (2.5), one finds that

$$
\begin{aligned}
\hat{h}_{0} & =E_{6} h_{0}, \\
\hat{h}_{\frac{1}{2}} & =E_{6} h_{\frac{1}{2}}, \\
\hat{h}_{\frac{i}{2}} & =E_{6} h_{\frac{i}{2}}, \\
\hat{h}_{\frac{1+i}{2}} & =E_{6} h_{\frac{1+i}{2}} .
\end{aligned}
$$

Hence $\psi_{12,1}^{+}=E_{6} \phi_{6,1}^{-}$. Observe that the modular Eisenstein series $E_{6}$ can also be viewed as a weight 6 and index 0 Hermitian Jacobi form of negative parity. We conclude that $\phi_{6,1}^{-} \in J_{6,1}^{-}$. 
We end this subsection with the initial Fourier series expansions of the Hermitian Jacobi forms $\phi_{4,1}^{+}, \phi_{6,1}^{-}, \phi_{8,1}^{+}$, and $\phi_{10,1}^{+, \text {cusp }}$ (for more coefficients of these forms, see [24]).

Remark 2.5. We have the following initial Fourier series expansions:

$$
\begin{aligned}
& \phi_{4,1}^{+}=1+q\left(60+32\left(\zeta^{\frac{1}{2}}\left(\zeta^{\prime}\right)^{\frac{1}{2}}+\zeta^{-\frac{1}{2}}\left(\zeta^{\prime}\right)^{-\frac{1}{2}}+\zeta^{-\frac{i}{2}}\left(\zeta^{\prime}\right)^{\frac{i}{2}}+\zeta^{\frac{i}{2}}\left(\zeta^{\prime}\right)^{-\frac{i}{2}}\right)\right. \\
& +\left(\zeta \zeta^{\prime}+\zeta^{-1}\left(\zeta^{\prime}\right)^{-1}+\zeta^{-i}\left(\zeta^{\prime}\right)^{i}+\zeta^{i}\left(\zeta^{\prime}\right)^{-i}\right) \\
& \left.+12\left(\zeta^{\frac{1+i}{2}}\left(\zeta^{\prime}\right)^{\frac{1-i}{2}}+\zeta^{\frac{-1+i}{2}}\left(\zeta^{\prime}\right)^{\frac{-1-i}{2}}+\zeta^{\frac{1-i}{2}}\left(\zeta^{\prime}\right)^{\frac{1+i}{2}}+\zeta^{\frac{-1-i}{2}}\left(\zeta^{\prime}\right)^{\frac{-1+i}{2}}\right)\right) \\
& +\cdots, \\
& \phi_{6,1}^{-}=1+q\left(-204-64\left(\zeta^{\frac{1}{2}}\left(\zeta^{\prime}\right)^{\frac{1}{2}}+\zeta^{-\frac{1}{2}}\left(\zeta^{\prime}\right)^{-\frac{1}{2}}+\zeta^{-\frac{i}{2}}\left(\zeta^{\prime}\right)^{\frac{i}{2}}+\zeta^{\frac{i}{2}}\left(\zeta^{\prime}\right)^{-\frac{i}{2}}\right)\right. \\
& +\left(\zeta \zeta^{\prime}+\zeta^{-1}\left(\zeta^{\prime}\right)^{-1}+\zeta^{-i}\left(\zeta^{\prime}\right)^{i}+\zeta^{i}\left(\zeta^{\prime}\right)^{-i}\right) \\
& \left.-12\left(\zeta^{\frac{1+i}{2}}\left(\zeta^{\prime}\right)^{\frac{1-i}{2}}+\zeta^{\frac{-1+i}{2}}\left(\zeta^{\prime}\right)^{\frac{-1-i}{2}}+\zeta^{\frac{1-i}{2}}\left(\zeta^{\prime}\right)^{\frac{1+i}{2}}+\zeta^{\frac{-1-i}{2}}\left(\zeta^{\prime}\right)^{\frac{-1+i}{2}}\right)\right) \\
& +\cdots, \\
& \phi_{8,1}^{+}=1+q\left(364+\left(\zeta \zeta^{\prime}+\zeta^{-1}\left(\zeta^{\prime}\right)^{-1}+\zeta^{-i}\left(\zeta^{\prime}\right)^{i}+\zeta^{i}\left(\zeta^{\prime}\right)^{-i}\right)\right. \\
& \left.+28\left(\zeta^{\frac{1+i}{2}}\left(\zeta^{\prime}\right)^{\frac{1-i}{2}}+\zeta^{\frac{-1+i}{2}}\left(\zeta^{\prime}\right)^{\frac{-1-i}{2}}+\zeta^{\frac{1-i}{2}}\left(\zeta^{\prime}\right)^{\frac{1+i}{2}}+\zeta^{\frac{-1-i}{2}}\left(\zeta^{\prime}\right)^{\frac{-1+i}{2}}\right)\right) \\
& +\cdots, \\
& \phi_{10,1}^{+, \text {cusp }}=q\left(\zeta^{\frac{1}{2}}\left(\zeta^{\prime}\right)^{\frac{1}{2}}+\zeta^{-\frac{1}{2}}\left(\zeta^{\prime}\right)^{-\frac{1}{2}}-\zeta^{-\frac{i}{2}}\left(\zeta^{\prime}\right)^{\frac{i}{2}}-\zeta^{\frac{i}{2}}\left(\zeta^{\prime}\right)^{-\frac{i}{2}}\right) \\
& +q^{2}\left(-18\left(\zeta^{\frac{1}{2}}\left(\zeta^{\prime}\right)^{\frac{1}{2}}+\zeta^{-\frac{1}{2}}\left(\zeta^{\prime}\right)^{-\frac{1}{2}}-\zeta^{-\frac{i}{2}}\left(\zeta^{\prime}\right)^{\frac{i}{2}}-\zeta^{\frac{i}{2}}\left(\zeta^{\prime}\right)^{-\frac{i}{2}}\right)\right. \\
& +\zeta^{\frac{1+2 i}{2}}\left(\zeta^{\prime}\right)^{\frac{1-2 i}{2}}+\zeta^{\frac{-1+2 i}{2}}\left(\zeta^{\prime}\right)^{\frac{-1-2 i}{2}}+\zeta^{\frac{1-2 i}{2}}\left(\zeta^{\prime}\right)^{\frac{1+2 i}{2}}+\zeta^{\frac{-1-2 i}{2}}\left(\zeta^{\prime}\right)^{\frac{-1+2 i}{2}} \\
& \left.-\zeta^{\frac{2+i}{2}}\left(\zeta^{\prime}\right)^{\frac{2-i}{2}}-\zeta^{\frac{-2+i}{2}}\left(\zeta^{\prime}\right)^{\frac{-2-i}{2}}-\zeta^{\frac{2-i}{2}}\left(\zeta^{\prime}\right)^{\frac{2+i}{2}}-\zeta^{\frac{-2-i}{2}}\left(\zeta^{\prime}\right)^{\frac{-2+i}{2}}\right)+\cdots \text {. }
\end{aligned}
$$

2.3. Proof of Theorem 1.1. We proceed as in 23] (see also $\S 3$ of [8]) to prove Theorem 1.1. Consider the Taylor series of a Hermitian Jacobi form $\phi \in J_{k, 1}^{\delta}$ around $(z, w)=(0,0)$ :

$$
\phi(\tau, z, w)=\sum_{\mu, \nu=0}^{\infty} \chi_{\mu, \nu}^{\delta}(\tau) z^{\mu} w^{\nu} .
$$

Then $\chi_{\mu, \nu}^{\delta}=0$ unless $\mu-\nu$ is even, and if $\epsilon\left(\begin{array}{ll}a & b \\ c & d\end{array}\right) \in \Gamma(\mathcal{O})$, then one finds that

$$
\begin{aligned}
& \chi_{\mu, \nu}^{\delta}\left(\frac{a \tau+b}{c \tau+d}\right) \\
& \begin{aligned}
=\sigma(\varepsilon) \varepsilon^{k-\mu+\nu}(c \tau+d)^{k+\mu+\nu} & \left(\chi_{\mu, \nu}^{\delta}(\tau)+\frac{2 \pi i c}{c \tau+d} \chi_{\mu-1, \nu-1}^{\delta}(\tau)\right. \\
& \left.\quad+\frac{1}{2 !}\left(\frac{2 \pi i c}{c \tau+d}\right)^{2} \chi_{\mu-2, \nu-2}^{\delta}(\tau)+\ldots\right) .
\end{aligned}
\end{aligned}
$$

The two following propositions on the Taylor coefficients $\chi_{\mu, \nu}^{\delta}$ are easy to verify, and we omit their proofs (see also [24]).

Proposition 2.6. Let $\phi(\tau, z, w)=\sum_{\mu, \nu=0}^{\infty} \chi_{\mu, \nu}^{\delta}(\tau) z^{\mu} w^{\nu} \in J_{k, 1}^{\delta}$.

If $k \equiv 0(\bmod 4)$ and $\delta=+$, then $\chi_{0,2}^{+}(\tau)=\chi_{2,0}^{+}(\tau)=0$. 
If $k \equiv 0(\bmod 4)$ and $\delta=-$, then $\chi_{0,0}^{-}(\tau)=\chi_{1,1}^{-}(\tau)=\chi_{4,0}^{-}(\tau)=\chi_{0,4}^{-}(\tau)=$ $\chi_{2,2}^{-}(\tau)=0$.

If $k \equiv 2(\bmod 4)$ and $\delta=-$, then $\chi_{0,2}^{-}(\tau)=\chi_{2,0}^{-}(\tau)=0$.

If $k \equiv 2(\bmod 4)$ and $\delta=+$, then $\chi_{0,0}^{+}(\tau)=\chi_{1,1}^{+}(\tau)=\chi_{4,0}^{+}(\tau)=\chi_{0,4}^{+}(\tau)=$ $\chi_{2,2}^{+}(\tau)=0$.

Proposition 2.7. Let $\phi(\tau, z, w)=\sum_{\mu, \nu=0}^{\infty} \chi_{\mu, \nu}^{\delta}(\tau) z^{\mu} w^{\nu} \in J_{k, 1}^{\delta}$. Then

$$
\begin{aligned}
& \chi_{0,0}^{\delta}(\tau) \in M_{k}, \\
& \chi_{2,0}^{\delta}(\tau), \chi_{0,2}^{\delta}(\tau) \in S_{k+2}, \\
& \chi_{4,0}^{\delta}(\tau), \chi_{0,4}^{\delta}(\tau) \in S_{k+4}, \\
& \zeta_{1,1}^{\delta}(\tau):=\chi_{1,1}^{\delta}(\tau)-\frac{2 \pi i}{k}\left(\chi_{0,0}^{\delta}\right)^{\prime}(\tau) \in S_{k+2}, \\
& \zeta_{2,2}^{\delta}(\tau):=\chi_{2,2}^{\delta}(\tau)-\frac{2 \pi i}{k+2}\left(\chi_{1,1}^{\delta}\right)^{\prime}(\tau)+\frac{(2 \pi i)^{2}}{2(k+1)(k+2)}\left(\chi_{0,0}^{\delta}\right)^{\prime \prime}(\tau) \in S_{k+4} .
\end{aligned}
$$

Remember the definition of the Jacobi theta function $\theta_{a, b}$ in (2.2) to verify the factorization

$$
\theta_{1, \frac{a+b i}{2}}^{H}(\tau, z, w)=\theta_{\frac{a}{2}, 0}(2 \tau, z+w) \theta_{\frac{b}{2}, 0}(2 \tau, i(w-z)) .
$$

Let $\phi \in J_{k, 1}^{\delta}$. Expand the theta decomposition

$$
\begin{aligned}
\phi(\tau, z, w) & =\sum_{a, b \in\{0,1\}} h_{\frac{a+b i}{2}}(\tau) \theta_{1, \frac{a+b i}{2}}^{H}(\tau, z, w) \\
& =\sum_{a, b \in\{0,1\}} h_{\frac{a+b i}{2}}(\tau) \theta_{\frac{a}{2}, 0}(2 \tau, z+w) \theta_{\frac{b}{2}, 0}(2 \tau, i(w-z))
\end{aligned}
$$

into a Taylor series, and compare its coefficients with the coefficients of the Taylor series

$$
\begin{aligned}
\phi(\tau, z, w)=\chi_{0,0}^{\delta}(\tau) & +\chi_{1,1}^{\delta}(\tau) z w+\left(\chi_{0,2}^{\delta}(\tau)+\chi_{2,0}^{\delta}(\tau)\right)\left(z^{2}+w^{2}\right)+\chi_{2,2}^{\delta}(\tau) z^{2} w^{2} \\
& +\left(\chi_{0,4}^{\delta}(\tau)+\chi_{4,0}^{\delta}(\tau)\right)\left(z^{4}+w^{4}\right)+\cdots .
\end{aligned}
$$

A direct calculation reveals that (see 24] for more details)

$$
\left(\chi_{0,0}^{\delta}, \chi_{1,1}^{\delta}, \chi_{2,0}^{\delta}, \frac{1}{2}\left(\chi_{2,2}^{\delta}-12 \chi_{4,0}^{\delta}\right)\right)=\left(h_{0}, h_{\frac{1}{2}}, h_{\frac{i}{2}}, h_{\frac{1+i}{2}}\right) A,
$$

where

$$
A:=\left(\begin{array}{cccc}
T_{0}{ }^{2} & 2 T_{0} T_{0}{ }^{\prime} & 0 & T_{0}{ }^{2} \\
T_{0} T_{1} & T_{0} T_{1}{ }^{\prime}+T_{0}{ }^{\prime} T_{1} & \frac{1}{4}\left(T_{1}{ }^{\prime} T_{0}-T_{1} T_{0}{ }^{\prime}\right) & 2 T_{0}{ }^{\prime} T_{1}{ }^{\prime} \\
T_{0} T_{1} & T_{0} T_{1}{ }^{\prime}+T_{0}{ }^{\prime} T_{1} & -\frac{1}{4}\left(T_{1}^{\prime} T_{0}-T_{1} T_{0}{ }^{\prime}\right) & 2 T_{0}{ }^{\prime} T_{1}{ }^{\prime} \\
T_{1}{ }^{2} & 2 T_{1} T_{1}{ }^{\prime} & 0 & T_{1}{ }^{2}
\end{array}\right)
$$

with

$$
T_{2 a}:=\theta_{a, 0}(2 \tau), \quad T_{2 a}{ }^{\prime}:=2 \pi i \frac{d}{d \tau} \theta_{a, 0}(2 \tau), \quad T_{2 a}{ }^{\prime \prime}:=(2 \pi i)^{2} \frac{d^{2}}{d \tau^{2}} \theta_{a, 0}(2 \tau),
$$

and where $T_{2 b}, T_{2 b}{ }^{\prime}$, and $T_{2 b}{ }^{\prime \prime}$ are defined analogously. Moreover, one finds that

$$
\operatorname{det} A=-\frac{1}{2}\left(T_{1} T_{0}{ }^{\prime}-T_{0} T_{1}{ }^{\prime}\right)^{2}\left(\left(T_{1} T_{0}{ }^{\prime}\right)^{2}-4 T_{0} T_{0}{ }^{\prime} T_{1} T_{1}{ }^{\prime}\right) \neq 0 .
$$

Now we are in a position to prove Theorem 1.1. We only consider the case $k \equiv 0(\bmod 4)$, since the proof of the case $k \equiv 2(\bmod 4)$ is completely analogous. 
Note that Proposition 2.7 shows that the map $\zeta$ is well-defined. First, we demonstrate that $\zeta$ is injective. Let $\phi=\left(\phi^{+}, \phi^{-}\right) \in J_{k, 1}$ such that $\zeta(\phi)=0$. Then $\chi_{0,0}^{+}=\zeta_{1,1}^{+}=\chi_{2,0}^{-}=\zeta_{2,2}^{+}-12 \chi_{4,0}^{+}=0$. Proposition 2.7 implies that $\chi_{1,1}^{+}=0$, $\zeta_{2,2}^{+}=\chi_{2,2}^{+}$, and hence $\frac{1}{2}\left(\chi_{2,2}^{+}-12 \chi_{4,0}^{+}\right)=0$. Furthermore, Proposition 2.6 gives that $\chi_{2,0}^{+}=0$ and $\chi_{0,0}^{-}=\chi_{1,1}^{-}=\chi_{2,2}^{-}=\chi_{4,0}^{-}=0$. Thus, for $\delta= \pm$ we have

$$
(0,0,0,0)=\left(\chi_{0,0}^{\delta}, \chi_{1,1}^{\delta}, \chi_{2,0}^{\delta}, \frac{1}{2}\left(\chi_{2,2}^{\delta}-12 \chi_{4,0}^{\delta}\right)\right) \underset{[2.7}{=}\left(h_{0}, h_{\frac{1}{2}}, h_{\frac{i}{2}}, h_{\frac{1+i}{2}}\right) A,
$$

where $A$ is as in (2.8). Since $\operatorname{det} A \neq 0$, we obtain $\phi=0$.

Next we show the injectivity of $\eta$. Let $(e, f, g, h) \in M_{k-4} \oplus M_{k-6} \oplus M_{k-8} \oplus M_{k-10}$ such that $e \phi_{4,1}^{+}+f \phi_{6,1}^{-}+g \phi_{8,1}^{+}+h \phi_{10,1}^{+, \text {cusp }}=0$. Observe the theta decompositions of $\phi_{4,1}^{+}, \phi_{8,1}^{+}$, and $\phi_{10,1}^{+, \text {cusp }}$ in (2.5) and of $\phi_{6,1}^{-}$in Lemma 2.4 to discover that

$$
(e, f, g, h) H=(0,0,0,0),
$$

where

$$
H:=\left(\begin{array}{cccc}
\frac{1}{2}\left(x^{6}+y^{6}\right) & \frac{1}{2} z^{6} & \frac{1}{2} z^{6} & \frac{1}{2}\left(x^{6}-y^{6}\right) \\
h_{0} & h_{\frac{1}{2}} & h_{\frac{i}{2}} & h_{\frac{1+i}{2}} \\
\frac{1}{2}\left(x^{14}+y^{14}\right) & \frac{1}{2} z^{14} & \frac{1}{2} z^{14} & \frac{1}{2}\left(x^{14}-y^{14}\right) \\
0 & \frac{1}{64} x^{6} y^{6} z^{6} & -\frac{1}{64} x^{6} y^{6} z^{6} & 0
\end{array}\right)
$$

with $h_{0}, h_{\frac{1}{2}}, h_{\frac{i}{2}}$, and $h_{\frac{1+i}{2}}$ as in Lemma 2.4. One finds that (note that $x^{4}=y^{4}+z^{4}$ )

$$
\operatorname{det} H=-\frac{9}{128} x^{16} y^{16} z^{16} \neq 0,
$$

which shows that $e=f=g=h=0$.

Finally,

$$
\begin{aligned}
& \operatorname{dim} M_{k}+\operatorname{dim} S_{k+2}+\operatorname{dim} S_{k+2}+\operatorname{dim} S_{k+4} \\
& \quad=\operatorname{dim} M_{k-4}+\operatorname{dim} M_{k-6}+\operatorname{dim} M_{k-8}+\operatorname{dim} M_{k-10},
\end{aligned}
$$

and we conclude that $\zeta$ and $\eta$ are isomorphisms.

Remark 2.8. The proof of Lemma 2.4 reveals that $E_{6} \phi_{6,1}^{-}=-2 E_{4}^{2} \phi_{4,1}^{+}+\frac{15}{2} E_{4} \phi_{8,1}^{+}-$ $\frac{9}{2} \phi_{12,1}^{+}$. Thus, the restriction of the maps $\eta$ and $\zeta$ in Theorem 1.1 to the case of positive parity yields the structure of $J_{k, 1}^{+}$in $[23$.

\section{Hermitian Jacobi forms modulo $p$}

3.1. Congruences and filtrations. In this section, we explore congruences and filtrations of Hermitian Jacobi forms. In particular, we establish the necessary tools to prove Theorem 1.2

For Hermitian Jacobi forms $\phi(\tau, z, w)=\sum c(n, r) q^{n} \zeta^{\bar{r}}\left(\zeta^{\prime}\right)^{r}$ and $\psi(\tau, z, w)=$ $\sum c^{\prime}(n, r) q^{n} \zeta^{\bar{r}}\left(\zeta^{\prime}\right)^{r}$ with $p$-integral rational coefficients, we write $\phi \equiv \psi(\bmod p)$ whenever $c(n, r) \equiv c^{\prime}(n, r)(\bmod p)$ for all $n, r$.

Proposition 3.1. If $\phi \in J_{k, 1}^{\delta}\left(\mathbb{Z}_{(p)}\right)$ such that $\phi=e \phi_{4,1}^{+}+f \phi_{6,1}^{-}+g \phi_{8,1}^{+}$(resp. $\phi=h \phi_{10,1}^{+, c u s p}$ ), then the elliptic modular forms $e, f$, and $g$ (resp. $h$ ) have p-integral rational coefficients.

Moreover, if $\phi \equiv 0(\bmod p)$, then $e \equiv f \equiv g \equiv 0(\bmod p)(\operatorname{resp} . h \equiv 0(\bmod p))$. 
Proof. The initial Fourier series expansions in Remark 2.5]imply that the generators $\phi_{4,1}^{+}, \phi_{6,1}^{-}, \phi_{8,1}^{+}$, and $\phi_{10,1}^{+, \text {cusp }}$ are linearly independent over the field $\mathbb{Z} / p \mathbb{Z}$ (see [24] for more details).

Suppose that $\phi=e \phi_{4,1}^{+}+f \phi_{6,1}^{-}+g \phi_{8,1}^{+}$(the case $\phi=h \phi_{10,1}^{+, \text {cusp }}$ is analogous). Note that the elliptic modular forms $e, f$, and $g$ have bounded denominators. If $e, f$, or $g$ do not have $p$-integral rational coefficients, then there exists some integer $t \geq 1$ such that $0 \equiv p^{t} \phi \equiv p^{t} e \phi_{4,1}^{+}+p^{t} f \phi_{6,1}^{-}+p^{t} g \phi_{8,1}^{+}(\bmod p)$. This yields a nontrivial linear dependence relation for $\phi_{4,1}^{+}, \phi_{6,1}^{-}$, and $\phi_{8,1}^{+}$, which contradicts the above.

Similarly, if $\phi \equiv 0(\bmod p)$ such that $e, f$, or $g$ do not vanish modulo $p$, then one also obtains a nontrivial linear dependence relation for $\phi_{4,1}^{+}, \phi_{6,1}^{-}$, and $\phi_{8,1}^{+}$, which is again a contradiction.

An argument as in the proof of Lemma 2.1 of Sofer [26] shows that if two Hermitian Jacobi forms of indices $m$ and $m^{\prime}$ are congruent modulo $p$, then $m=m^{\prime}$. We now give an analog of Sofer's Lemma 2.1 in the case $m=1$.

Corollary 3.2. Let $\phi \in J_{k, 1}^{\delta}\left(\mathbb{Z}_{(p)}\right)$ and $\psi \in J_{k^{\prime}, 1}^{\delta^{\prime}}\left(\mathbb{Z}_{(p)}\right)$ such that $0 \not \equiv \phi \equiv$ $\psi(\bmod p)$. Then $k \equiv k^{\prime}(\bmod (p-1))$.

Proof. Recall that if two elliptic modular forms $f_{i} \in M_{k_{i}}(i=1,2)$ have $p$-integral rational coefficients such that $0 \not \equiv f_{1} \equiv f_{2}(\bmod p)$, then $k_{1} \equiv k_{2}(\bmod (p-1))($ see [25,28]). This fact in combination with Proposition 3.1 implies the claim.

We also record the following consequence of Theorem 1.1 and Proposition 3.1 .

Corollary 3.3. Let $\phi \in J_{k, 1}^{\delta}\left(\mathbb{Z}_{(p)}\right)$ and $\psi \in J_{k^{\prime}, 1}^{\delta^{\prime}}\left(\mathbb{Z}_{(p)}\right)$ such that $\phi \equiv \psi(\bmod p)$. If $\delta \neq \delta^{\prime}$ and $k \equiv k^{\prime}(\bmod 4)$, then $\phi \equiv \psi \equiv 0(\bmod p)$.

Corollary 3.2 shows that there are congruences among Hermitian Jacobi forms of different weights, and one wishes to find the smallest weight in which the (coefficientwise) reduction of a Hermitian Jacobi form modulo $p$ exists.

Definition 3.4. Set $\widetilde{J_{k, 1}^{\delta}}:=\left\{\phi(\bmod p): \phi \in J_{k, 1}^{\delta}\left(\mathbb{Z}_{(p)}\right)\right\}$. For Hermitian Jacobi forms with $p$-integral rational coefficients, we define the filtration modulo $p$ by

$$
\Omega(\phi):=\inf \left\{k: \phi(\bmod p) \in \widetilde{J_{k, 1}^{\delta}}\right\} .
$$

Next we generalize Proposition 2 of 22] (see also Proposition 2.15 of [20]) to the case of Hermitian Jacobi forms of index 1.

Proposition 3.5. If $\phi \in J_{k, 1}^{\delta}\left(\mathbb{Z}_{(p)}\right)$, then $L(\phi)(\bmod p)$ is the reduction of a Hermitian Jacobi form modulo $p$. Moreover, we have

$$
\Omega(L(\phi)) \leq \Omega(\phi)+p+1,
$$

with equality if and only if $p \nmid \Omega(\phi)-1$.

Proof. It is easy to adapt the proofs in 20,22 to the case of Hermitian Jacobi forms. Specifically, one employs (1.2), Proposition 3.1. Corollary 3.2, and Theorem 2 and Lemma 5 of [28]. We omit further details, which are contained in [24].

Tate's theory of theta cycles (see $\S 7$ of 13 ) was extended to Jacobi forms (see [21]) and Jacobi forms of higher degree (see [20]). The arguments in [20,21] apply also to Hermitian Jacobi forms, and Corollary 3.2 and Proposition 3.5 are the key ingredients in proving Theorem 1.2. We omit the detailed proof of Theorem 1.2 , which is contained in 24 . 
3.2. An example. As in the case of elliptic modular forms, one does not know if a given Hermitian Jacobi form has $U(p)$ congruences for only finitely many primes. In the following example, we consider primes $5 \leq p<100$, and we show that $E_{4}^{2} \phi_{10,1}^{+, \text {cusp }} \in J_{18,1}^{+}$has $U(p)$ congruences for $p=5,7,13,23,79$, and for no other primes $5 \leq p<100$ (see 24] for more examples). If $p \neq 5,7,13,23,79$, then the table of Fourier series coefficients of $E_{4}^{2} \phi_{10,1}^{+, \text {cusp }}$ in the Appendix of [24] guarantees that $E_{4}^{2} \phi_{10,1}^{+, c u s p} \mid U(p) \not \equiv 0(\bmod p)$.

Recall the Ramanujan theta operator $\Theta:=q \frac{d}{d q}=\frac{1}{2 \pi i} \frac{d}{d \tau}$, and Ramanujan's [19] identities (up to a factor of 4 )

$$
\begin{aligned}
& L\left(E_{2}\right)=4 \Theta\left(E_{2}\right)=\frac{1}{3}\left(E_{2}^{2}-E_{4}\right), \\
& L\left(E_{4}\right)=4 \Theta\left(E_{4}\right)=\frac{4}{3}\left(E_{2} E_{4}-E_{6}\right), \\
& L\left(E_{6}\right)=4 \Theta\left(E_{6}\right)=2\left(E_{2} E_{6}-E_{4}^{2}\right) .
\end{aligned}
$$

Moreover, (1.2) together with Theorem 1.1 and Remark 2.5 yields the following identities

$$
\begin{aligned}
L\left(\phi_{4,1}^{+}\right) & =E_{2} \phi_{4,1}^{+}-\phi_{6,1}^{-}, \\
L\left(\phi_{6,1}^{-}\right) & =\frac{5}{3} E_{2} \phi_{6,1}^{-}-\frac{8}{3} E_{4} \phi_{4,1}^{+}+\phi_{8,1}^{+}, \\
L\left(\phi_{8,1}^{+}\right) & =\frac{7}{3} E_{2} \phi_{8,1}^{+}-\frac{14}{9} E_{6} \phi_{4,1}^{+}-\frac{7}{9} E_{4} \phi_{6,1}^{-}, \\
L\left(\phi_{10,1}^{+, \text {cusp }}\right) & =3 E_{2} \phi_{10,1}^{+, \text {cusp }} .
\end{aligned}
$$

We employ (3.1) and (3.2) in combination with the well-known congruences $E_{p-1} \equiv$ $1(\bmod p)$ and $E_{p+1} \equiv E_{2}(\bmod p)$ to establish (with the help of Mathematica) the $U(p)$ congruences for $E_{4}^{2} \phi_{10,1}^{+, c u s p}$ for $p=5,7,13,23,79$.

Let $p=5,7$, or 13. We cannot apply Theorem 1.2 since $p<18$. However, straightforward calculations show that $L^{p-1}\left(E_{4}^{2} \phi_{10,1}^{+, c u s p}\right) \equiv E_{4}^{2} \phi_{10,1}^{+, c u s p}(\bmod p)$, which implies the desired $U(p)$ congruences.

If $p=23$ or 79 , then we apply Theorem 1.2 .

Let $p=23$. One finds that $E_{22} \equiv 10 E_{4}^{4} E_{6}+14 E_{4} E_{6}^{3} \equiv 1(\bmod 23)$. Moreover, $p+2-18=7$, and a direct calculation reveals that

$$
L^{7}\left(E_{4}^{2} \phi_{10,1}^{+, \text {cusp }}\right) \equiv\left(20 E_{4}^{4} E_{6}+5 E_{4} E_{6}^{3}\right) \phi_{10,1}^{+, \text {cusp }} \equiv 2 E_{22} \phi_{10,1}^{+, \text {cusp }} \equiv 2 \phi_{10,1}^{+, \text {cusp }}(\bmod 23) .
$$

Hence $\Omega\left(L^{7}\left(E_{4}^{2} \phi_{10,1}^{+, \text {cusp }}\right)\right)=10=p+5-18$, and $E_{4}^{2} \phi_{10,1}^{+, \text {cusp }} \mid U(23) \equiv 0(\bmod 23)$.

Let $p=79$. One finds that

$$
\begin{aligned}
E_{78} & \equiv 26 E_{4}^{18} E_{6}+10 E_{4}^{15} E_{6}^{3}+73 E_{4}^{12} E_{6}^{5}+33 E_{4}^{9} E_{6}^{7}+41 E_{4}^{6} E_{6}^{9}+72 E_{4}^{3} E_{6}^{11}+62 E_{6}^{13} \\
& \equiv 1(\bmod 79) .
\end{aligned}
$$

Moreover, $p+2-18=63$, and a direct calculation shows that

$$
\begin{aligned}
& L^{63}\left(E_{4}^{2} \phi_{10,1}^{+, c u s p}\right) \\
& \equiv\left(73 E_{4}^{32} E_{6}+46 E_{4}^{29} E_{6}^{3}+70 E_{4}^{26} E_{6}^{5}+12 E_{4}^{23} E_{6}^{7}+57 E_{4}^{20} E_{6}^{9}+75 E_{4}^{17} E_{6}^{11}\right. \\
& \left.+61 E_{4}^{14} E_{6}^{13}+9 E_{4}^{11} E_{6}^{15}+16 E_{4}^{8} E_{6}^{17}+39 E_{4}^{5} E_{6}^{19}+31 E_{4}^{2} E_{6}^{21}\right) \phi_{10,1}^{+, \text {cusp }} \\
& \equiv E_{78}\left(18 E_{4}^{14}+7 E_{4}^{11} E_{6}^{2}+71 E_{4}^{8} E_{6}^{4}+37 E_{4}^{5} E_{6}^{6}+40 E_{4}^{2} E_{6}^{8}\right) \phi_{10,1}^{+, \text {cusp }} \\
& \equiv\left(18 E_{4}^{14}+7 E_{4}^{11} E_{6}^{2}+71 E_{4}^{8} E_{6}^{4}+37 E_{4}^{5} E_{6}^{6}+40 E_{4}^{2} E_{6}^{8}\right) \phi_{10,1}^{+, c u s p}(\bmod 79) \text {. }
\end{aligned}
$$

Hence $\Omega\left(L^{63}\left(E_{4}^{2} \phi_{10,1}^{+, \text {cusp }}\right)\right)=66=79+5-18$, and $E_{4}^{2} \phi_{10,1}^{+, \text {cusp }} \mid U(79) \equiv 0(\bmod 79)$. 


\section{ACKNOWLEDGMENTS}

The authors thank Martin Raum for several useful discussions, and for writing the SAGE code that allowed them to determine the Hermitian Jacobi form $\phi_{6,1}^{-}$ in Lemma 2.4.

\section{REFERENCES}

[1] Scott Ahlgren and Ken Ono, Arithmetic of singular moduli and class polynomials, Compos. Math. 141 (2005), no. 2, 293-312, DOI 10.1112/S0010437X04001198. MR.2134268 (2006a:11058)

[2] Anatolii N. Andrianov, Modular descent and the Saito-Kurokawa conjecture, Invent. Math. 53 (1979), no. 3, 267-280, DOI 10.1007/BF01389767. MR549402 (80k:10024)

[3] YoungJu Choie, Jacobi forms and the heat operator, Math. Z. 225 (1997), no. 1, 95-101, DOI 10.1007/PL00004603. MR 1451334 (98c:11042)

[4] YoungJu Choie, Jacobi forms and the heat operator. II, Illinois J. Math. 42 (1998), no. 2, 179-186. MR1612731 (99d:11049)

[5] Soumya Das, Note on Hermitian Jacobi forms, Tsukuba J. Math. 34 (2010), no. 1, 59-78. MR2723724 (2012b:11077)

[6] Soumya Das, Some aspects of Hermitian Jacobi forms, Arch. Math. (Basel) 95 (2010), no. 5, 423-437, DOI 10.1007/s00013-010-0176-3. MR2738862 (2012a:11055)

[7] T. Dern, Hermitesche Modulformen zweiten Grades, Ph.D. thesis, RWTH Aachen University, Germany, 2001.

[8] Martin Eichler and Don Zagier, The theory of Jacobi forms, Progress in Mathematics, vol. 55, Birkhäuser Boston, Inc., Boston, MA, 1985. MR781735 (86j:11043)

[9] Noam Elkies, Ken Ono, and Tonghai Yang, Reduction of CM elliptic curves and modular function congruences, Int. Math. Res. Not. 44 (2005), 2695-2707, DOI 10.1155/IMRN.2005.2695. MR2181309 (2006k:11076)

[10] Klaus Haverkamp, Hermitesche Jacobiformen (German), Schriftenreihe des Mathematischen Instituts der Universität Münster. 3. Serie, Vol. 15, Schriftenreihe Math. Inst. Univ. Münster 3. Ser., vol. 15, Univ. Münster, Münster, 1995, pp. 105. MR1335247 (96c:11053)

[11] Klaus K. Haverkamp, Hermitian Jacobi forms, Results Math. 29 (1996), no. 1-2, 78-89, DOI 10.1007/BF03322207. MR1377681|(97d:11077)

[12] Jun-ichi Igusa, On the graded ring of theta-constants, Amer. J. Math. 86 (1964), 219-246. MR0164967 (29 \#2258)

[13] Naomi Jochnowitz, A study of the local components of the Hecke algebra mod l, Trans. Amer. Math. Soc. 270 (1982), no. 1, 253-267, DOI 10.2307/1999771. MR.642340 (83e:10033a)

[14] Haesuk Kim, Differential operators on Hermitian Jacobi forms, Arch. Math. (Basel) 79 (2002), no. 3, 208-215, DOI 10.1007/s00013-002-8306-1. MR1933379 (2003g:11047)

[15] Hans Maass, Über eine Spezialschar von Modulformen zweiten Grades (German), Invent. Math. 52 (1979), no. 1, 95-104, DOI 10.1007/BF01389857. MR/532746 (80f:10031)

[16] Hans Maass, Über eine Spezialschar von Modulformen zweiten Grades. II (German), Invent. Math. 53 (1979), no. 3, 249-253, DOI 10.1007/BF01389765. MR549400(81a:10037)

[17] Hans Maass, Über eine Spezialschar von Modulformen zweiten Grades. III (German), Invent. Math. 53 (1979), no. 3, 255-265, DOI 10.1007/BF01389766. MR549401 (81a:10038)

[18] Ken Ono, The web of modularity: arithmetic of the coefficients of modular forms and q-series, CBMS Regional Conference Series in Mathematics, vol. 102, Published for the Conference Board of the Mathematical Sciences, Washington, DC by the American Mathematical Society, Providence, RI, 2004. MR2020489 (2005c:11053)

[19] S. Ramanujan, On certain arithmetical functions, Trans. Camb. Phil. Soc. 22 (1916), 159184, (Collected Papers, No. 18).

[20] M. Raum and O. Richter, The structure of Siegel modular forms mod $p$ and $U(p)$ congruences, to appear in Mathematical Research Letters.

[21] Olav K. Richter, On congruences of Jacobi forms, Proc. Amer. Math. Soc. 136 (2008), no. 8, 2729-2734, DOI 10.1090/S0002-9939-08-09274-5. MR2399034(2009i:11060)

[22] Olav K. Richter, The action of the heat operator on Jacobi forms, Proc. Amer. Math. Soc. 137 (2009), no. 3, 869-875, DOI 10.1090/S0002-9939-08-09566-X. MR2457425|(2010i:11064) 
[23] Ryuji Sasaki, Hermitian Jacobi forms of index one, Tsukuba J. Math. 31 (2007), no. 2, 301-325. MR2371175 (2008j:11055)

[24] J. Senadheera, Hermitian Jacobi forms and congruences, Ph.D. thesis, University of North Texas, 2014.

[25] Jean-Pierre Serre, Formes modulaires et fonctions zêta p-adiques (French), Modular functions of one variable, III (Proc. Internat. Summer School, Univ. Antwerp, 1972), Springer, Berlin, 1973, pp. 191-268. Lecture Notes in Math., Vol. 350. MR0404145 (53 \#7949a)

[26] Adriana Sofer, p-adic aspects of Jacobi forms, J. Number Theory 63 (1997), no. 2, 191-202, DOI 10.1006/jnth.1997.2095. MR 1443756(98b:11058)

[27] W. A. Stein et al., Sage Mathematics Software (Version 5.7), The Sage Development Team, 2013, http://www.sagemath.org.

[28] H. P. F. Swinnerton-Dyer, On l-adic representations and congruences for coefficients of modular forms, Modular functions of one variable, III (Proc. Internat. Summer School, Univ. Antwerp, 1972), Springer, Berlin, 1973, pp. 1-55. Lecture Notes in Math., Vol. 350. MR.0406931(53 \#10717a)

[29] D. Zagier, Sur la conjecture de Saito-Kurokawa (d'après H. Maass) (French), Seminar on Number Theory, Paris 1979-80, Progr. Math., vol. 12, Birkhäuser, Boston, Mass., 1981, pp. 371-394. MR633910 (83b:10031)

Department of Mathematics, University of North Texas, Denton, Texas 76203

E-mail address: richter@unt.edu

Department of Mathematics, University of North Texas, Denton, Texas 76203

E-mail address: JayanthaSenadheera@my.unt.edu

Current address: Department of Mathematics and Computer Science, Faculty of Natural Sciences, The Open University of Sri Lanka, Nawala 10250, Sri Lanka 